\title{
CARACTERÍSTICAS LABORALES Y SOCIOECONÓMICAS DE LOS ESTUDIANTES DE LA FACULTAD DE CIENCIAS CONTABLES
}

\author{
LABOR AND SOCIO-ECONOMIC CHARACTERISTICS OF STUDENTS \\ OF THE FACULTY OF ACCOUNTING \\ Domingo Guzmán Chumpitaz Ramos * \\ Docente Auxiliar de la Facultad de Ciencias Contables \\ Héctor Félix Cerna Maguiña ** \\ Docente Asociado de la Facultad de Ciencias Contables \\ Universidad Nacional Mayor de San Marcos-UNMSM / Lima-Perú \\ [Recepción: Marzo de 2014/ Conformidad: Mayo 2014]
}

\section{RESUMEN}

El presente trabajo de investigación tiene como finalidad documentar y proporcionar información a nuestras autoridades, docentes, administrativos y estudiantes sobre las características laborales y socioeconómicas de los estudiantes del último año de la Facultad de Ciencias Contables de la UNMSM - 2013. Este estudio tiene un enfoque cualitativo, de tipo de investigación descriptiva. La población, fueron los estudiantes del último año de la facultad. Se tomó una muestra de 186 estudiantes, aplicándose un muestreo estratificado. El instrumento que utilizamos fue un cuestionario de 36 preguntas.

Se presentan los resultados respecto a las características personales y educativas de los estudiantes, tales como: genero, edad, residencia, idioma, aspectos de elección de la universidad y de la carrera profesional de Contabilidad. También se tiene información valiosa sobre las características laborales y económicas de los estudiantes como: si trabajan actualmente, ingreso, tipo de empresa en la que trabajan, así como, su desempeño laboral.

La principal conclusión del trabajo de investigación, es la existencia de una independencia entre las características laborales del tipo de ocupación y las características económicas del rango de sus salarios. Los salarios de los estudiantes varían respecto al tipo de ocupación que desempeñan.

\section{Palabras Clave:}

Estadística descriptiva; variable; validez; confiabilidad; cuestionario; gráficos; análisis.

\begin{abstract}
This research work aims to document and provide information to our Authorities, Teachers, Administrative and Student employment and socioeconomic characteristics of the final year students of the School of Accounting Sciences UNMSM-2013. This study has a qualitative approach, descriptive research type. The population were seniors in college. A sample of 186 students was taken, applying stratified sampling. The instrument used was a questionnaire of 36 questions. Results are presented regarding personal and educational characteristics of students, such as gender, age, residence, language, aspects of choice of college and career Accounting. It also has valuable information on labor and economic characteristics of students as if it works currently, income, type of company you work for, as well as their job performance. The main conclusion of the research is the existence of an independence between work characteristics of the type of occupation and the economic characteristics of the range of wages. Wages of students vary in the type of occupation they perform.
\end{abstract}

\section{Keywords:}

Descriptive statistics; Variable; validity; reliability; questionnaire; graphics; analysis.

\footnotetext{
* Licenciado en Estadística. Estadístico Colegiado. Email: dgchr95@hotmail.com

** Magister en Docencia Universitaria, Lic. Investigación Operativa, Colegiado en el COMAP. Email: hector_fcm@hotmail.com
} 


\section{INTRODUCCIÓN}

La Facultad de Ciencias Contables de la UNMSM demanda en sus estudiantes una aplicación con las máximas exigencias, características propias de la profesión. El estudiante de la carrera logra formarse como un profesional competente en el mercado laboral.

El presente trabajo titulado, "Características Laborales y Socioeconómicas de los Estudiantes de pregrado del último año de la Facultad de Ciencias Contables de la UNMSM (2013)", busca actualizar la información sobre la población estudiantil de la facultad, llevando a cabo estudios sobre fenómenos educativos, laborales y socioeconómicos, con el ánimo de servir de apoyo a las diferentes carreras y dependencias de la Institución, facilitando la toma de decisiones con información actualizada y pertinente, siempre en la búsqueda del mejoramiento continuo en el quehacer formador e institucional; teniendo siempre como objetivo final, preparar a personas idóneas que den respuesta a las necesidades propias y del entorno.

Por lo tanto, es recomendable que lo señalado en el trabajo sirva de referencia a las autoridades académicas y administrativas de la universidad, que tengan un vínculo directo con los servicios a los estudiantes. A su vez, también se convierte en una fuente de información valiosa, para entidades externas que quieran saber más sobre aspectos laborales y socioeconómicos de los estudiantes universitarios.

Este tipo de estudio nace de la necesidad de identificar cuál es el origen socioeconómico de los estudiantes de la facultad, a efectos de ofrecerles las mejores opciones y servicios universitarios y garanticen su satisfacción con la formación recibida. Cualquier política que afecte de alguna forma a los estudiantes de la institución, necesariamente debería tener en cuenta los aspectos que en este estudio se consignan, ya que solo en la medida en que se conozcan y se entiendan las características que identifican a los estudiantes de la Facultad de Ciencias Contables, en aspectos sociales, económicos, familiares, académicos y laborales, se podrán generar los cambios que den mejores respuestas y se acoplen a las necesidades de los estudiantes y su grupo familiar.

Para realizar esta investigación se hizo un trabajo de campo, teniendo como instrumento un cuestionario, el mismo que fue aplicado solamente a los estudiantes del último año de la facultad. Después de la recolección de información, obtuvimos datos, donde realizamos un análisis descriptivo que nos ha permitido tomar decisiones a futuro sobre la demanda laboral de los estudiantes de nuestra facultad.

La investigación, fue reforzada por los software estadísticos como el SPSS, Minitab, Stata y el R; de las cuales se obtuvo los cuadros y gráficos estadísticos, para luego analizarlos e interpretarlos.

\section{FUNDAMENTACIÓN DEL PROBLEMA}

El presente trabajo, surge a razón de la preocupación de saber si los estudiantes de los últimos ciclos de estudio de la Facultad de Ciencias Contables, se encuentran desempeñando labores de acuerdo al tipo de ocupación y al género. Por lo tanto, se formula la pregunta: ¿Cuál es la relación entre las características laborales y socioeconómicas de los estudiantes?

Los resultados de la investigación brindarán información valiosa con el fin de tomar decisiones de protección al futuro profesional y propender a que el estudiante y egresado en particular, se encuentre en puestos laborales relacionados con su formación académica, a fin de evitar el subempleo.

\section{OBJETIVO GENERAL}

Determinar la relación entre las características laborales y características socioeconómicas de los estudiantes del último año de estudios de pregrado de la Facultad de Contabilidad de la UNMSM.

\section{OBJETIVOS ESPECÍFICOS}

- Describir el género, promedio de edad, lugar de nacimiento, lugar de residencia y estado civil de los estudiantes del último año de la Facultad de Ciencias Contables.

- Describir las características académicas; el idioma, tipos de institución donde terminó la educación secundaria, tipo de preparación para postular a esta universidad, modalidad de ingreso, aspecto principal que influyó para elegir esta universidad de los estudiantes del último año de la Facultad de Ciencias Contables. 
- Describir las características laborales; de prácticas pre-profesionales, si trabaja actualmente, actividad de la empresa en la que trabaja y desempeño ocupacional en su trabajo de los estudiantes del último año de la Facultad de Ciencias Contables.

- Describir las características económicas; ingreso monetario de su ocupación principal y horas de trabajo al día de los estudiantes del último año de la Facultad de Ciencias Contables.

- Medir el grado de relación del ingreso monetario según su ocupación principal.

\section{RESULTADOS DEL ESTUDIO}

El 2013, en la Facultad de Ciencias Contables de la UNMSM, han egresado más estudiantes del sexo femenino (59.68\%) que del sexo masculino
(40.32\%). El promedio de edad es de 23.27 años. El $82.26 \%$ de estudiantes son naturales de Lima.

El $60.75 \%$ provienen de un centro educativo público. Y el 33.33\% provienen de una institución particular. Del total de estudiantes del último año de la Facultad de Ciencias Contables en el 2013, se prepararon para ingresar el 53.23\% en una Academia; mientras que a través del Centro Pre-Universitario, ingresaron el $39.78 \%$ y, el resto ingresó preparándose por su cuenta y con ayuda de un profesor particular.

En el gráfico $\mathrm{N}^{\circ} 1$, observamos que el idioma Inglés es el más preferido por los estudiantes del último año, el 62.2\% lo escribe-lee-habla. Existe poca demanda de preferencia de los estudiantes, respecto a los otros idiomas.

El 94.58\% del total conoce el idioma Inglés.

\section{Gráfico $\mathrm{N}^{\circ} 1$ \\ Idioma según modalidad}

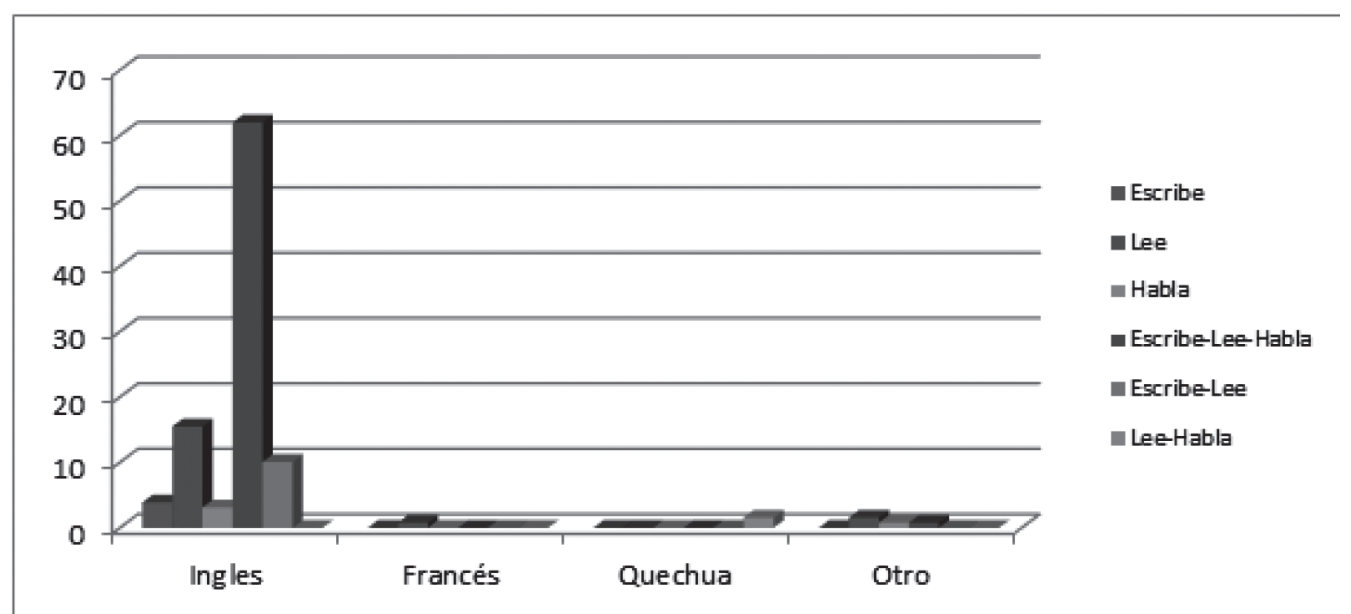

Fuente y elaboración: Propia.

En relación a su carrera profesional el estudiante consiguió su Prácticas Pre- profesionales por su cuenta, con un $81.25 \%$. En el gráfico $\mathrm{N}^{\circ} 2$ observamos que el $94 \%$ de estudiantes entrevistados trabajan, mientras que el $6 \%$ no trabajan, solamente se dedican a estudiar. El 78.86\% del total de estudiantes trabajan en el sector de administración y servicios. A proximadamente el 18\% trabajan en el sector de industrias y muy pocos estudiantes, trabajan en los sectores de pesca, agricultura, ganadería, etcétera.

\section{Gráfico $\mathrm{N}^{\circ} 2$}

Estudiantes que trabajan

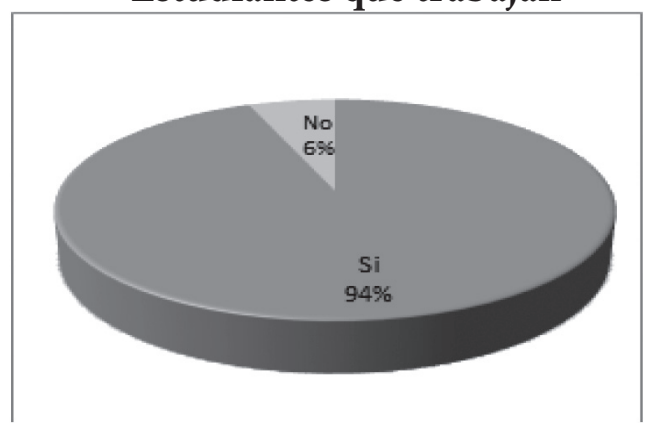

Fuente y elaboración: Propia. 
En el gráfico $\mathrm{N}^{\circ}$ 3, observamos que el $50.9 \%$ de estudiantes que han egresado de la Facultad de Contabilidad se desempeñan como Asistentes Contables; el $19.4 \%$ se desempeñan en su trabajo como Auxiliar Contable y como Analista Contable trabajan aproximadamente el 5\%. En otras ocupaciones están el $25.1 \%$ de estudiantes. Respecto a los tipos de ocupaciones siempre hay una marcada diferencia.

Gráfico $\mathrm{N}^{\circ} 3$ Ingresos por rangos

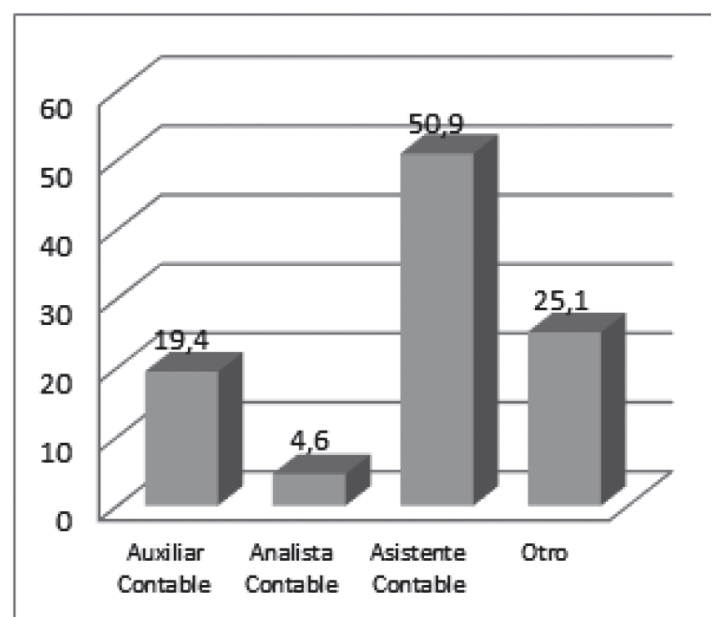

Fuente y elaboración: Propia.

En el gráfico $\mathrm{N}^{\circ} 4$, observamos que el $40 \%$ del total de estudiantes ganan entre 1001 y 1500 nuevos soles. Entre 501 y 1000 nuevos soles ganan el 30.9\% del total. La mayoría de estudiantes trabajan 8 horas al día.

\section{Gráfico $\mathrm{N}^{\circ} 4$ \\ Tipo de ocupación}

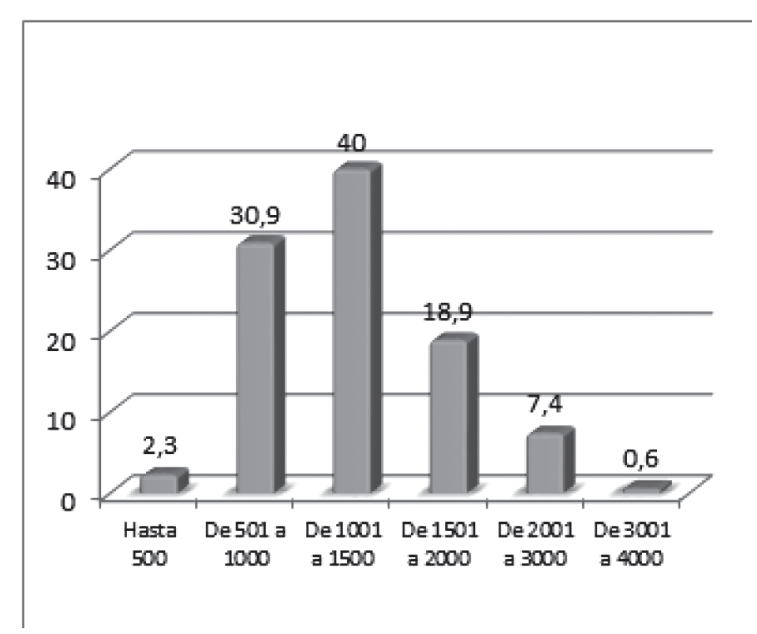

Fuente y elaboración: Propia.

En el grafico $\mathrm{N}^{\circ} 5$, observamos que el $2.36 \%$ de estudiantes con ocupación de Asistentes Contables ganan salarios superiores a 2000 nuevos soles, mientras que no existen estudiantes que trabajen como Analista o Auxiliar, pero el 3.4\% de estudiantes se dedican a otro tipo de trabajo. Entre 1000 y 2000 nuevos soles es el ingreso de los Asistentes Contables con $35.4 \%$ del total de estudiantes entrevistados, se diferencia mucho de las otras ocupaciones. Concluimos que hay diferencias en el tipo de ocupación respecto a cada rango de ingreso, eso significa que no hay relación entre estas dos variables.

\section{Gráfico $\mathrm{N}^{\circ} 5$ \\ Ingreso según tipo de ocupación}

Auxiliar Contable Analista Contable Asistente Contable @ Otro

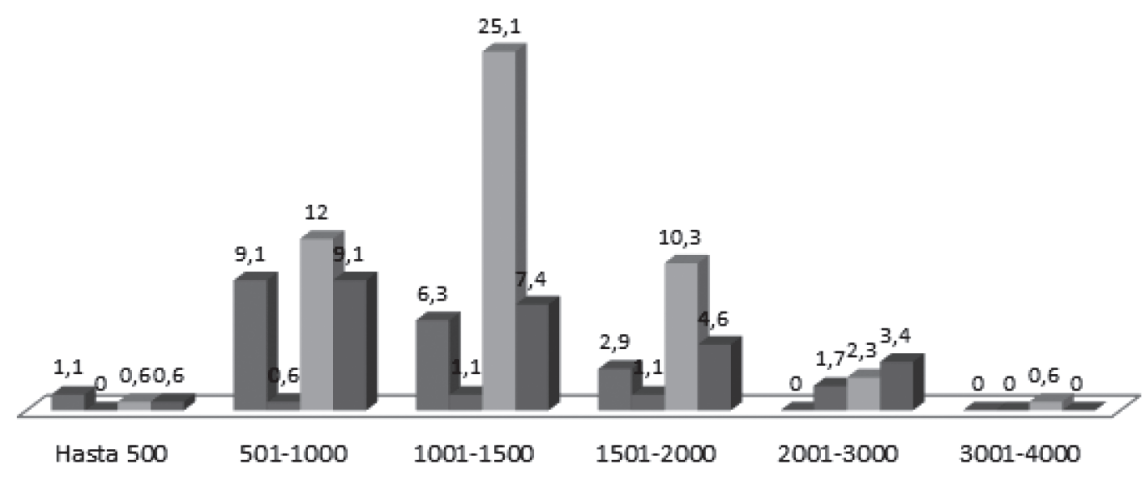

Fuente y elaboración: Propia. 


\section{CONCLUSIONES}

1. Respecto a las características personales de los estudiantes, en la Facultad de Contabilidad de la UNMSM, han egresado en el 2013 más mujeres que hombres, mayormente los estudiantes son limeños.

2. Respecto a las características académicas de los estudiantes que han egresado de la Facultad de Contabilidad en el 2013, la mayoría dominan el idioma inglés y provienen de colegios públicos. Se han preparado en academias pre-universitarias y han ingresado por examen de admisión optando postular a la UNMSM por prestigio.

3. Respecto a las características laborales-económicas del estudiante de la Facultad de Contabilidad, la mayoría ha realizado sus Prácticas Pre-profesionales; casi todos trabajan, se desempeñan como administrativos y a su vez como Asistentes Contables.

4. Respecto a las características económicas; se concluye que el sueldo de la mayoría varía entre 1000 y 2000 nuevos soles y trabajan 8 horas diarias.

\section{RECOMENDACIONES}

1. Se recomienda a las autoridades académicas y administrativas de la Facultad de Ciencias Contables de la UNMSM mejorar la calidad de servicio y formación académica del estudiante para el cumplimiento de sus objetivos profesionales y sus metas trazadas.

2. La Facultad de Ciencias Contables debe ser implementada y equipada con todo lo necesario para brindar las condiciones en el logro de enseñanza-aprendizaje de calidad.
3. La Facultad de Ciencias Contables debe contar con convenios interinstitucionales ya sean públicos y privados para mejorar las condiciones académicas.

\section{REFERENCIAS BIBLIOGRÁFICAS}

1. WELKOWITZ, J., Ewen R. y Jacob Cohen J. (1981) - "Estadística Aplicada a las Ciencias de la Educación” Madrid Santilla, aula XXI.

2. Figuera, P. (1996) "La inserción del universitario en el mercado de trabajo”. Barcelona: EUB.

\section{REFERENCIAS HEMEROGRÁFICAS}

1. González García, L. (1993). "Nuevas relaciones entre educación, trabajo y empleo en la década de los 90"; Revista Iberoamericana de Educación $\mathrm{N}^{\circ} 2$.

2. Rodríguez Lajo, M., Vila, R. y Freixa, M. (2008). "Barreras de género y actitudes hacia las redes sociales en alumnado universitario de las Facultades de Educación". Revista de Investigación Educativa, 26 (1), 45-72.

3. GALTUNG, Johan (1978). “Teoría y métodos de la investigación Social” Tomos I y y volumen 1.

4. Trinidad Donoso Vázquez, Pilar Figuera Gazo, Ma Luisa Rodríguez Moreno (2009). "Modelos Sociales y Mercado Laboral: Un Estudio con Alumnado Universitario" Revista de Ciencias Sociales $\mathrm{N}^{\circ} 2$.

\section{REFERENCIAS ELECTRÓNICAS}

1. Caro Sánchez Y. (2010). Caracterización de los Estudiantes del Pre-Grado de la Facultad de Medicina de la Universidad Antioquia, http://www.alfaguia.org/alfaguia/files/1322019116_2777.pdf 